\title{
National Breast Cancer Mortality and Incidence Rates According to the Human Development Index: An Ecological Study
}

\author{
Salman Khazaei', Shahab Rezaeian'2, Zaher Khazaei ${ }^{3}$, Leila Molaeipoor ${ }^{4}$, \\ Shahrzad Nematollahi ${ }^{*}$, Parvaneh Lak ${ }^{6}$, Somayeh Khazaei ${ }^{7}$ \\ ${ }^{1}$ Department of Epidemiology, School of Public Health, Hamadan University of Medical Sciences, Hamadan, \\ Iran \\ ${ }^{2}$ Social Development \& Health Promotion Research Center, Gonabad University of Medical Sciences, Gonabad, \\ Iran \\ ${ }^{3}$ Social Determinants of Health Research Center, Kurdistan University of Medical Sciences, Sanandaj, Iran \\ ${ }^{4}$ Department of Epidemiology, Pasteur Institute of Iran, Tehran, Iran \\ ${ }^{5}$ Department of Epidemiology and Biostatistics, School of Public Health, Tehran University of Medical Sciences, \\ Tehran, Iran \\ ${ }^{6} \mathrm{MD}$ of Obs \& Gyn, Shaheed Chamran Hospital, Iran University of Medical Sciences, Tehran, Iran \\ ${ }^{7}$ BSc in Operating Room, Rafsanjan University of Medical Sciences, Rafsanjan, Iran \\ Email: salmankhazaei61@gmail.com, shahab.rezayan@gmail.com, Zaherkhazaei@yahoo.com, \\ Leilamolaei_epi@yahoo.com, "shahrzadnema@yahoo.com, lakparvaneh@yahoo.com
}

Received 12 December 2015; accepted 24 January 2016; published 27 January 2016

Copyright (C 2016 by authors and Scientific Research Publishing Inc.

This work is licensed under the Creative Commons Attribution International License (CC BY).

http://creativecommons.org/licenses/by/4.0/

\section{(c) (i) Open Access}

\begin{abstract}
Background: This study aimed to identify the role of human development index (HDI) in the incidence and mortality rates of breast cancer (BC) worldwide. Methods: Data on the age-standardized incidence and mortality rates of BC for 184 countries were obtained from the GLOBOCAN. Data about the HDI and other indices were obtained from the World Bank Report 2013. Linear regression model was used for assessment the effect of HDI on BC occurrence rates. Results: In 2012, BCs were estimated to have affected a total of 1,671,149 individuals (crude rate: 47.8 per 100,000 individuals), and caused 521,907 deaths worldwide (crude rate: 14.7 per 100,000 individuals). Nearly half of total female BC cases $(46.3 \%)$ with the highest risk of incidence (age-standardized Rate (ASR): 128 per 100,000) had occurred in very high HDI regions. The most proportion of the mortality burden was in low HDI and medium HDI areas. Linear regression analyses showed a direct significant correlation between the incidence of $B C$ and $H D I$ at the global level $(B=104.5, P<$ $0.001)$. The mortality rate of $B C$ was not significantly associated with $H D I(B=3.26, P=0.160)$.
\end{abstract}

${ }^{*}$ Corresponding author.

How to cite this paper: Khazaei, S., Rezaeian, S., Khazaei, Z., Molaeipoor, L., Nematollahi, S., Lak, P. and Khazaei, S. (2016) National Breast Cancer Mortality and Incidence Rates According to the Human Development Index: An Ecological Study. Advances in Breast Cancer Research, 5, 30-36. http://dx.doi.org/10.4236/abcr.2016.51003 
Conclusion: Our study showed that the burden of female BC is enormous in very high HDI and low HID regions. Targeted interventions have the ability to reduce this number significantly through resource-dependent interventions. Moreover, further reductions in mortality could be brought about by increasing access to curative treatment for patients with BC.

\title{
Keywords
}

\author{
Breast Cancer, Human Development Index, Incidence, Age-Standardized Incidence and Mortality \\ Rates
}

\section{Introduction}

Cancer, already the leading cause of death in many high-income countries, is set to become a major cause of morbidity and mortality in the rest of the world irrespective of level of resources [1]. Breast cancer (BC) is regarded as the most common health problem in women worldwide. In 2012, BC was considered as the fifth cause of cancer death, having caused 1.67 million new cases which accounted for $25 \%$ of all new cancer cases in the world [2]. BC has been occurred in substantial variation ranging from 27 cases in Middle Africa and Eastern Asia to 96 cases in Western Europe countries (per 100,000 population) [3]. Every year, BC causes 324,000 deaths (14.3\% of total cancer deaths in these countries) in underprivileged societies, and 198,000 deaths (15.4\% of total cancer deaths in these countries) in privileged ones [3].

Changing patterns of demographic, social, and economical aspects of life has led to changes in reproductive patterns, ageing and increases of life-expectancy in most regions of the world. Therefore, cancers associated with infectious causes have been replaced by cancers associated with life style factors such as diet, and hormonal factors [4] [5]. Despite the high incidence in developed countries, BC mortality imposes the greater burden in developing world due to urbanizations, westernization, and increased life expectancy [6] [7], as well as unequal distribution of resources such as access to medical care, physician distribution; and psychological factors such as stress, or lack of social support [8]-[11] as important related factors have been reported.

$\mathrm{BC}$ has become a prominent cause of morbidity and mortality all around the world, necessitating the tendency towards incorporation of the socio-economic status into diagnostic measures. Having investigated several factors associated with BC, unequal distribution of Human Development Index (HDI) has been widely studied [12]-[14]. HDI is comprised of three components namely education, life expectancy and gross national income (GNI) with ranges between 0 to 1 , and is appropriate for defining socio-economic status of the societies. This index has categorized countries into four categories namely very high, high, medium, and low by linking geographical patterns of BC with corresponding levels of HDI. An overview of this type of cancer is provided in the present study. Our intention is to draw attention to variations in BC-specific rates in each HDI region.

\section{Methods}

This is an ecological study on the relation of the age-specific incidence and mortality rate (ASR) of BC and the HDI.

HDI has several main components that include: life expectancy at birth, mean years of schooling, and gross national income (GNI) per capita; and also some ancillary indexes including total fertility rate (number of children that would be born to a woman if she were to live to the end of her child-bearing years and bear children at each age in accordance with prevailing age-specific fertility rates), percent of urbanization, and age standardized obesity (defined as BMI > 30) in adults (the weighted average of the age-specific obesity rate among adults ages 20 and older). ASR is a summary measure of the rate that having a standard age structure, a population distribution would have since age has a powerful influence on the risk of cancer, standardization is necessary when comparing several populations that differ with respect to age.

Data of the ASR for 184 countries about incidence and mortality rate of BC for the year 2012 was obtained from the global cancer project available at http://globocan.iarc.fr/Default.aspx. GLOBOCAN also provided regional estimates for each continent. Data about the HDI and other indices of 169 countries were obtained from the World Bank Report 2013 available at http://databank.worldbank.org/data/reports.aspx. The analysis was re- 
stricted to 169 countries which both the epidemiologic data from the GLOBOCAN database and HDI were available. These countries categorized to: Very High Human Development (27 countries), High Human Development (37 countries), Medium Human Development (89 countries) and Low Human Development (16 countries). In this study, we used linear regression models for assessment of the HDI effect on BC occurrence rates. Multiple linear regression models were exempt due to extreme collinearity of predictors. The level of 0.05 was considered as statistical significance. Data were analyzed by Stata software version 12 (StataCorp, College Station, TX, USA).

\section{Results}

In 2012, BCs were estimated to have affected a total of 1,671,149 individuals (crude rate: 47.8 per 100,000 individuals), and caused 521,907 deaths worldwide (crude rate: 14.7 per 100,000 individuals). According to GLOBOCAN database, female BC was responsible for 36.3\% of total cancer cases (Figure 1).

The lifetime cumulative risk of female BC in very-high and high developed regions were as high as 78.2 and 45.2 cases per 100,000, respectively (Table 1 ). As development index decreases, the number of cases decreases as well. In this regard, female BC occurred in 26.5 cases (per 100,000 females) in medium developed, as well as 32.6 cases in low developed countries.

The highest cumulative risks are belonged to developed countries including Latin Americas, Europe and Australia. In addition, nearly half of total female BC cases (46.3\%) with the highest risk of incidence (age-standardized Rate (ASR): 128 per 100,000) in 2012 had occurred in very high HDI regions (Figure 2). A greater proportion of the mortality burden is seen in low HDI and medium HDI areas. Table 2 describes the different components of HDI along with occurrence of BC according to different development status of countries.

Using linear regression model, increment of HDI various components have all a positive effect on increase in incidence and mortality rates of BC. Specifically, increasing one unit in HDI can increase the mean of incidence rate by 104.5. The same conclusion can also be drawn for the impact of HDI on mortality risk. It can be seen that increasing one unit in HDI can cause increasing of mean mortality of BC by 3.26 though not significant. The increment of mean years of schooling had the strongest effect of significantly increasing mean incidence and mortality risk by 5.13 and 0.25 , respectively. Furthermore, while the impact of total fertility rate on increase in occurrence rates of $\mathrm{BC}$ was in opposite direction, only its impact on incidence rate yielded significant coefficient.

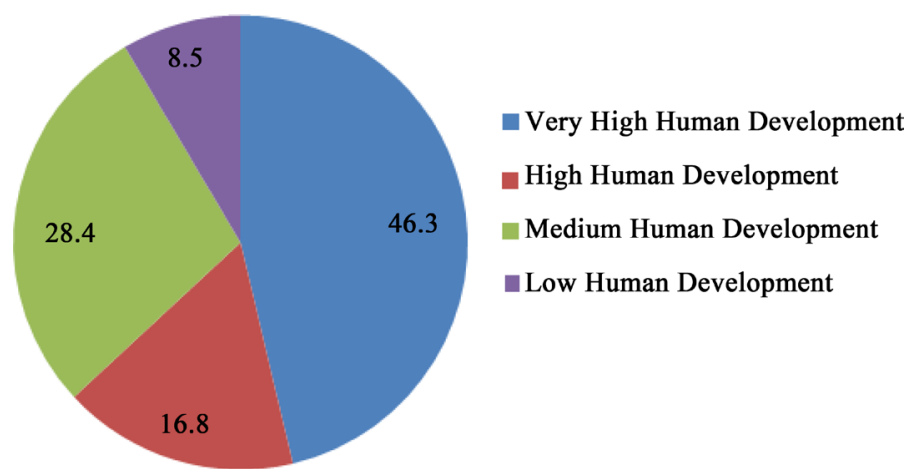

Figure 1. Percent of total breast cancer cases in adult population by HDI regions in 2012.

Table 1. Breast cancer incidence and mortality and HDI component by different HDI regions in 2012.

\begin{tabular}{|c|c|c|c|c|c|c|c|c|}
\hline \multirow{2}{*}{ Region } & \multicolumn{2}{|c|}{ Breast cancer incidence } & \multicolumn{3}{|c|}{ Breast cancer mortality } & \multicolumn{3}{|c|}{ HDI component } \\
\hline & CR & ASR & CR & ASR & LEB & MYS & GNI & HDI \\
\hline Very high human development & 128 & 78.2 & 29 & 14.1 & 80.2 & 11.7 & 40,046 & 0.89 \\
\hline High human development & 53.2 & 45.2 & 18 & 14.6 & 74.5 & 8.1 & 13,231 & 0.74 \\
\hline Medium human development & 28.5 & 26.5 & 10 & 9.8 & 67.9 & 5.5 & 5960 & 0.61 \\
\hline Low human development & 22.7 & 32.6 & 11.5 & 17 & 59.4 & 4.2 & 2904 & 0.49 \\
\hline
\end{tabular}

CR: Crude Rate, ASR: Age-Standardized Rates per 100,000, HDI: Human Development Index, LEB: Life Expectancy at Birth, MYS: Mean years of schooling, GNI: Gross national income per capita. 


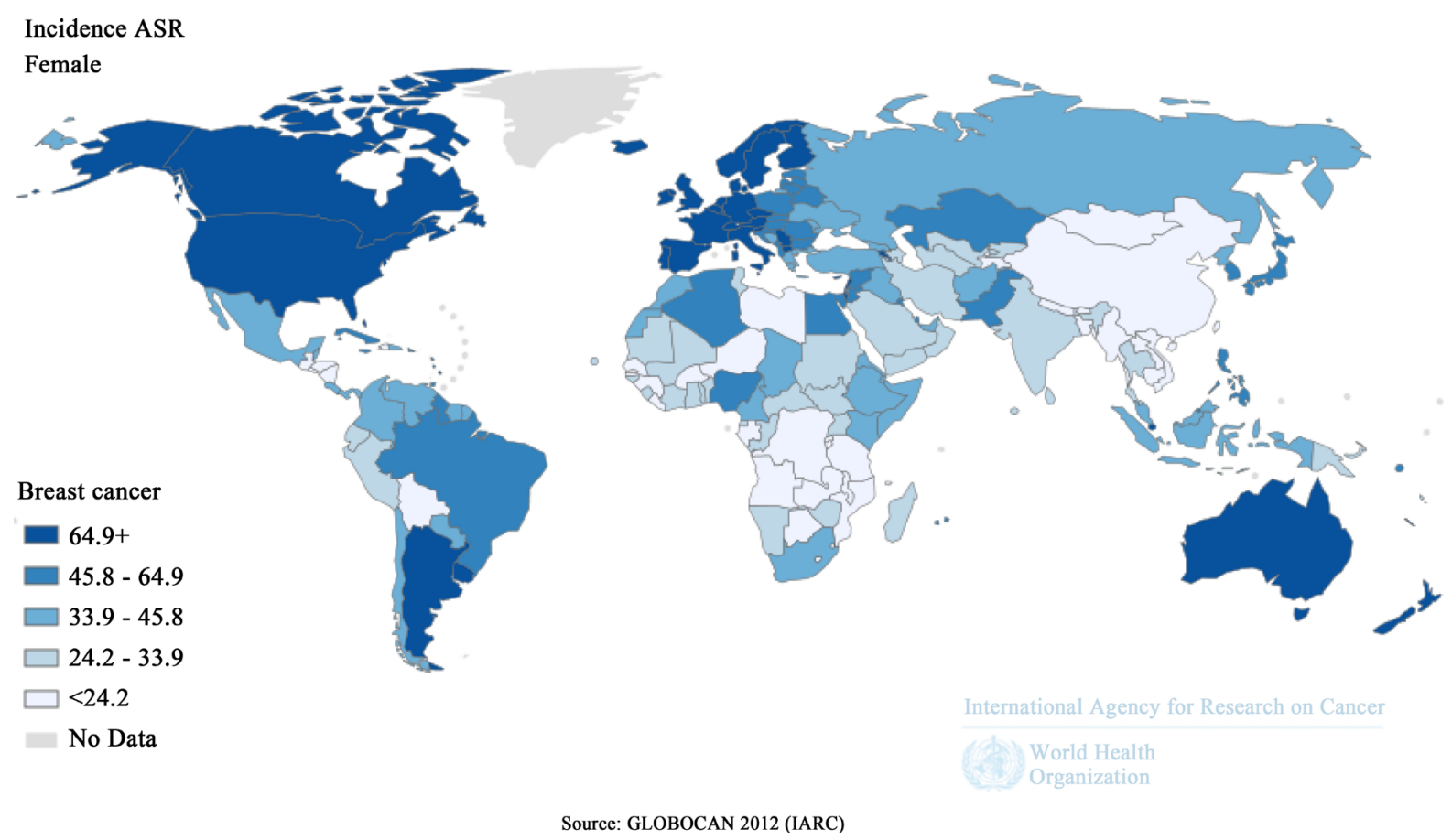

Figure 2. Incidence age-standardized rates per 100,000 of breast cancer in the world in 2012.

Table 2. Effect of HDI components and demographic variables on breast cancer incidence and mortality.

\begin{tabular}{ccccccc}
\hline \multirow{2}{*}{ Variable } & \multicolumn{3}{c}{ Breast cancer incidence } & \multicolumn{3}{c}{ Breast cancer mortality } \\
\cline { 2 - 7 } & B & CI & P-value & B & CI & P-value \\
\hline Life expectancy at birth & 1.67 & $1.37,1.97$ & $<0.001$ & 0.04 & $-0.037,0.12$ & 0.23 \\
Mean years of schooling & 5.13 & $4.3,5.97$ & $<0.001$ & 0.25 & $0.02,0.48$ & 0.033 \\
Gross national income per 1000 capita & 0.74 & $0.57,0.9$ & $<0.001$ & 0.01 & $-0.03,0.04$ & 0.66 \\
HDI & 104.5 & $87.7,120.2$ & $<0.001$ & 3.26 & $-1.3,7.8$ & 0.16 \\
Urbanization level (\%) & 0.57 & $0.45,0.7$ & $<0.001$ & -0.15 & $-0.65,0.34$ & 0.54 \\
Total fertility rate & -8.47 & $-10.5,-6.4$ & $<0.001$ & 0.02 & $-0.01,0.05$ & 0.27 \\
Age standardized obesity in adults & 0.97 & $0.66,1.27$ & $<0.001$ & 0.085 & $0.02,0.15$ & 0.014 \\
\hline
\end{tabular}

The impact of increasing age-standardized adulthood obesity on BC incidence and mortality risk was worthmention as well. Increasing one unit in age-standardized obesity in adults would resulted in significantly increased in mean incidence by 0.97 and in mean mortality by 0.08 .

\section{Discussion}

BC has been recognized as important reproductive health problem for women in both developed and developing countries [15]. Female BC accounts for about $46 \%$ of the cancer burden in very high and high, $29 \%$ in medium, and $9 \%$ in low HDI regions. Specifically, age-standardized incidence of female BC ranged from 78 in highdeveloped to 26.5 in medium-developed regions (per 100,000 women).

Research examining the correlation between macroeconomic determinants with BC incidence, and mortality; and how the relation varies by country are scarce. Assessment of these burden indicators against the specific components of HDI (education, health, and living standards) should enable examination of their relative importance [16]. Cancer is a heterogeneous mix of diseases. It has multiple causes, some of which are well understood and some of which are poorly understood. In addition, differences in the regional and temporal distribution of risk factors will determine the geographical and secular patterns of cancer. Availability of information about disease incidence and mortality, understanding of the underlying causes and how these can change, and know- 
ledge of the demographics of populations in terms of size and age structure can attenuate barriers of understanding the patterns of cancer [12].

In both high and very high HDI regions, female BC explain almost half the overall incidence burden. Incidence estimates will probably become more accurate by the expansion of high quality population-based cancer registries in low- and middle-income countries, as well as improvements and completeness of valid registrations in areas already covered. Similar to our results, Bray et.al has found that female BC is the most commonly diagnosed neoplasm in almost all countries [12]. Describing different components of HDI in these regions; it can be seen that all HDI components have been raised in very high developed regions which might make the high incidence of BC and the lower mortality of BC. This logic can be inferred for low developed region as well.

In medium HDI regions, age-standardized rate of incidence of female BC remains lowest. However, this type of cancer along with cancers caused by infectious agents is considered among highest health problems in these regions by other authors [17] [18]. Our study has shown that female BC is the only cancer that is common in all regions, irrespective of level of HDI. Among the vast majority of the world, it is regarded as the most common form of cancer in women, and thus the global control of BC through both early detection and primary prevention is a high priority [12] [13] [19]. Depend on the availability of data; a tendency towards increases in BC exists in the very high, high, and medium HDI regions. These effects are likely strongest in countries with very high HDI levels relative to those of medium or high HDI, which might be indicative of an incidence plateau having being reached. Nevertheless, the trends point to the westernization effect in countries with high HDI or medium HDI levels, and with increasing average levels of social and economic development. The population prevalence of several reproductive, dietary, metabolic, and hormonal risk factors could correspondingly increase the risk of these cancers.

This study attempted to incorporate recorded data to inform on the future cancer burden, but even more important step would be further appraisal which takes into account the specific preventative actions in the respective countries and regions of the world. The present situation is extrapolated recorded rates by use of crude averages of the estimated annual percentage change that are heterogeneous across registries in every HDI category, and thus should be interpreted with caution.

One clear concern is the reliance on extrapolation in only six population-based cancer registries (including two from China and India) to be representative of all medium-resource settings. Clearly, more data and greater precision are needed. Despite the unified modeling framework by 187 countries in the globe, comparisons of their modeled cumulative risk estimates (ages 15 - 79 years) of BC incidence showed a systematic overestimation in all countries [20].

In terms of the global burden predictions, the low HDI populations constitute $57 \%$ of the world's population and $16 \%$ of the cancer burden, and the absence of this information will have a minor effect [21]. The incidence and mortality estimates from GLOBOCAN are also subject to precision limitations. In the compilation of country-specific cancer incidence and mortality, a hierarchy of methods is used in GLOBOCAN which are relied on the quality and availability of the source information [12] [22]. More generally, African, Asian, and Latin Americas countries are suffered from high quality population-based cancer registries. Specifically, only $1 \%, 4 \%$, and $6 \%$ of the populations of these respective regions covered, and there are few if any national vital registration systems with adequate mortality data especially in Africa and Asia.

GLOBOCAN is a comprehensive data source for examination of the cancer burden worldwide and in the promotion of global cancer control. Nevertheless, population-based cancer registries have been shown to be a crucial component in national cancer control strategies, where the registered incidence information provides the tools to plan, monitor, and assess the effect of specific interventions in targeted populations [23].

\section{Conclusion}

Our study showed that the burden of female BC is enormous in very high HDI and low HID regions. Targeted interventions have the ability to reduce this number significantly through resource-dependent interventions. Such actions include primary prevention strategies to effectively control the prevalence of lifestyle factors including tobacco avoidance and cessation of smoking, a reduction in alcohol consumption and obesity, and the promotion of increased levels of physical activity; as well as the implementation of early detection programs. Moreover, further reductions in mortality could be brought about by increasing access to curative treatment for patients with BC. 


\section{Acknowledgements}

This paper used data from the GLOBOCAN and World Bank Report. The authors declare that there is no conflict of interests to report for this work.

\section{References}

[1] Ferlay, J., Shin, H.R., Bray, F., Forman, D., Mathers, C. and Parkin, D.M. (2010) Estimates of Worldwide Burden of Cancer in 2008: GLOBOCAN 2008. International Journal of Cancer, 127, 2893-2917. http://dx.doi.org/10.1002/ijc.25516

[2] World Health Organization (2015) Cancer. WHO, Geneva.

[3] International Agency for Research on Cancer (2012) GLOBACAN 2012: Estimated Breast Cancer Incidence, Mortality and Prevalence Worldwide in 2012. JARC. http://globocan.iarc.fr/Pages/fact_sheets_cancer.aspx

[4] The Cancer Atlas (2012) Human Development Index Transitions: As Countries Develop, Their Cancer Burden Changes in Scale and Type. http://canceratlas.cancer.org/the-burden/human-development-index-transitions/

[5] Rezaeian, S., Veisani, Y., Ghorbani, M., Delpisheh, A. and Abbastabar, H. (2015) Migraine History and Breast Cancer Risk: A Systematic Review and Meta-Analysis. Advances in Breast Cancer Research, 4, 63-70. http://dx.doi.org/10.4236/abcr.2015.43007

[6] Montazeri, A., Vahdaninia, M., Ebrahimi, M. and Jarvandi, S. (2003) The Hospital Anxiety and Depression Scale (HADS): Translation and Validation Study of the Iranian Version. Health and Quality of Life Outcomes, 1, 14. http://dx.doi.org/10.1186/1477-7525-1-14

[7] World Health Organization (2012) Breast Cancer: Prevention and Control. WHO, Geneva.

[8] Akinyemiju, T., Genkinger, J., Farhat, M., Wilson, A., Gary-Webb, T. and Tehranifar, P. (2015) Residential Environment and Breast Cancer Incidence and Mortality: A Systematic Review and Meta-Analysis. BMC Cancer, 15, 191. http://dx.doi.org/10.1186/s12885-015-1098-z

[9] Harper, S., Lynch, J., Meersman, S.C., Breen, N., Davis, W.W. and Reichman, M.C. (2009) Trends in Area-Socioeconomic and Race-Ethnic Disparities in Breast Cancer Incidence, Stage at Diagnosis, Screening, Mortality, and Survival among Women Ages 50 Years and over (1987-2005). Cancer Epidemiology Biomarkers \& Prevention, 18, 121131. http://dx.doi.org/10.1158/1055-9965.EPI-08-0679

[10] Xue, Q.Y. (2009) Socioeconomic Disparities in Breast Cancer Survival: Relation to Stage at Diagnosis, Treatment and Race. BMC Cancer, 9, 364. http://dx.doi.org/10.1186/1471-2407-9-364

[11] Mitchell, K.J., Fritschi, L., Reid, A., McEvoy, S.P., Ingram, D.M., Jamrozik, K., et al. (2006) Rural-Urban Differences in the Presentation, Management and Survival of Breast Cancer in Western Australia. The Breast, 15, 769-776. http://dx.doi.org/10.1016/j.breast.2006.04.001

[12] Bray, F., Jemal, A., Grey, N., Ferlay, J. and Forman, D. (2012) Global Cancer Transitions According to the Human Development Index (2008-2030): A Population-Based Study. The Lancet Oncology, 13, 790-801. http://dx.doi.org/10.1016/S1470-2045(12)70211-5

[13] Alwan, A., MacLean, D.R., Riley, L.M., d’Espaignet, E.T., Mathers, C.D., Stevens, G.A., et al. (2010) Monitoring and Surveillance of Chronic Non-Communicable Diseases: Progress and Capacity in High-Burden Countries. The Lancet, 376, 1861-1868. http://dx.doi.org/10.1016/S0140-6736(10)61853-3

[14] Ghoncheh, M., Mohammadian-Hafshejani, A. and Salehiniya, H. (2015) Incidence and Mortality of Breast Cancer and their Relationship to Development in Asia. Asian Pacific Journal of Cancer Prevention, 16, 6081-6087. http://dx.doi.org/10.7314/APJCP.2015.16.14.6081

[15] United Nations Population Fund (1995) Report of the International Conference on Population and Development. UNFPA, New York. http://www.unfpa.org/webdav/site/global/shared/documents/publications/2004/icpd_eng.pdf

[16] United Nations Development Programme (2011) Human Development Report. http://hdr.undp.org/en/data

[17] Polk, D.B. and Peek Jr., R.M. (2010) Helicobacter Pylori: Gastric Cancer and Beyond. Nature Reviews Cancer, 10, 403-414. http://dx.doi.org/10.1038/nrc2857

[18] Perz, J.F., Armstrong, G.L., Farrington, L.A., Hutin, Y.J. and Bell, B.P. (2006) The Contributions of Hepatitis B Virus and Hepatitis C Virus Infections to Cirrhosis and Primary Liver Cancer Worldwide. Journal of Hepatology, 45, 529538. http://dx.doi.org/10.1016/j.jhep.2006.05.013

[19] Farmer, P., Frenk, J., Knaul, F.M., Shulman, L.N., Alleyne, G., Armstrong, L., et al. (2010) Expansion of Cancer Care and Control in Countries of Low and Middle Income: A Call to Action. The Lancet, 376, 1186-1193. http://dx.doi.org/10.1016/S0140-6736(10)61152-X

[20] Ferlay, J., Forman, D., Mathers, C.D. and Bray, F. (2012) Breast and Cervical Cancer in 187 Countries between 1980 
and 2010: A Systematic Analysis. The Lancet, 379, 1390-1391. http://dx.doi.org/10.1016/S0140-6736(12)60595-9

[21] Jha, P. (2009) Avoidable Global Cancer Deaths and Total Deaths from Smoking. Nature Reviews Cancer, 9, 655-664. http://dx.doi.org/10.1038/nrc2703

[22] Myrskyla, M., Kohler, H.P. and Billari, F.C. (2009) Advances in Development Reverse Fertility Declines. Nature, 460, 741-743. http://dx.doi.org/10.1038/nature08230

[23] World Health Organization (2000) National Cancer Control Programmes: Policies and Managerial Guidelines. 2nd Edition, WHO, Geneva. 